\title{
In-Situ Analysis of Material Modifications During Deep Rolling by Synchrotron X-Ray Diffraction Experiments
}

\author{
Heiner Meyer ${ }^{1, a *}$ and Jérémy Epp ${ }^{1,2, b}$ \\ ${ }^{1}$ Leibniz Institute for Materials Engineering - IWT, Division Materials Science, Badgasteiner \\ Straße 3, 28359 Bremen, Germany \\ ${ }^{2}$ MAPEX Center for Materials and Processes, University of Bremen, Bibliothekstraße 1, 28359 \\ Bremen, Germany \\ ahmeyer@iwt-bremen.de, bepp@iwt-bremen.de
}

\begin{abstract}
Keywords: Deep Rolling, Synchrotron XRD, In-Situ X-Ray Diffraction, Cold Working, Process Signatures
\end{abstract}

\begin{abstract}
The stress state achieved through elasto-plastic deformation in the deep rolling process has been analyzed with in-situ synchrotron experiment using a roller as tool. For the determination of internal material load and residual stress for specific contact parameters, the material state was analyzed via transmission synchrotron X-ray diffraction for hundreds of measurement positions below the loaded zone. From the individual diffraction rings at each point, the strain and stress fields were reconstructed as 2D mappings of the material modifications. The measured region included material in front of the roller, under the contact point as well as in the already processed near surface region. A $13 \mathrm{~mm}$ cylindrical tungsten carbide roller was applied on specimens of AISI 4140 steel in ferritic-pearlitic and quenched and tempered condition to compare the effect of different mechanical properties on the propagation and intensity of the stress and strain fields in the specimen during and after processing. The investigations allowed a comparison of the stress fields for the different material conditions. The results show that the mechanisms of load distribution during the process and the effect of loading stresses on the final material state and on the residual stress distribution are strongly influenced by the initial material state.
\end{abstract}

\section{Introduction}

Residual stresses introduced into the surface through mechanically induced plastic deformation are important aspects of the process effects in deep rolling and roller burnishing. Analysis of surface residual stresses and of depth profiles generated by deep rolling is possible ex-situ after the process through standard methods such as X-Ray Diffraction (XRD), hole drilling techniques and other methods. It is well known that compressive residual stresses may be introduced several hundred micrometers into the material, depending on the process parameters, as shown by Meyer and Kaemmler [1]. These are connected with improved near surface hardness and an increased fatigue resistance for processed parts, as found by Nikitin and Altenberger [2]. The material response to the applied loading stresses and plastic deformation depends on the initial material parameters like yield strength, strain hardening behavior and microstructural stability, but while these parameters may be included in FEM simulations, as done by Sayahi et al. [3], the resulting loading stress and residual stress fields can show deviations from experimentally measured values, or may even be only accessible in-situ during the process. The connection between loading stresses and the resulting residual stresses is however most important to understand the process and its effect on different material states. This approach is used in the determination of process signatures to develop a mechanism-based prediction of the material modifications in

(c) (1) Content from this work may be used under the terms of the Creative Commons Attribution 3.0 license. Any further distribution of this work must maintain attribution to the author(s) and the title of the work, journal citation and DOI. Published under license by Materials Research Forum LLC. 
metal processing, as proposed by Brinksmeier et al. [4]. The information about loading stresses and the resulting residual stresses from mechanical elasto-plastic deformation can give insight into the material behavior and the modification mechanisms. To actually analyze the stress fields in-situ during processing, diffraction measurements using synchrotron radiation with sufficient penetration depth are an efficient method, as shown by Uhlmann et al. [5] for the analysis of an orthogonal cutting process.

In the present study, in-situ synchrotron XRD experiments were performed during deep rolling of steel grade AISI 4140 (42CrMo4) in two different heat treatment conditions (ferritepearlite and quenched + tempered). The aim was to analyze the material behavior under contact during applied pressure and movement to reconstruct 2D maps of stress state and peak width change from the measured diffraction signal. Based on these results, the material reaction during the process was evaluated in terms of cold working and generated residual stresses introduced for the two initial microstructures.

\section{Experimental methods}

For the experiments performed on beamline ID11 of the European Synchrotron Radiation Facility (ESRF), a transportable frame was used with a modified deep rolling tool, based on the system of Ecoroll AG, Celle, Germany. The tool head consist of a $13 \mathrm{~mm}$ diameter tungstencarbide cylinder of $15 \mathrm{~mm}$ width. Outfitted with a linear drive, specimens of dimension $70 \times 20 \times$ $2.8 \mathrm{~mm}(\mathrm{~L} \times \mathrm{H} \times \mathrm{W})$ were processed at a feed rate of $\mathrm{v}=0.02 \mathrm{~mm} / \mathrm{s}$, which can be considered as quasi-static process with low strain rate. Prior to the experiments, the surface was electropolished to a width of $2.8 \mathrm{~mm}$, to reduce influence from heat treatment and preprocessing steps on the measured material state. The cylindrical roller produces a consistent deep rolled state over the whole width of the surface. The force was applied via a hydraulic system at 350 bar which corresponds to a contact force of the tool of $3400( \pm 100) \mathrm{N}$. The setup, as installed in the beamline, is seen in Fig. 1a, while the transmission geometry measurement layout with FReLoN detector position is shown in the sketch in Fig.1b.

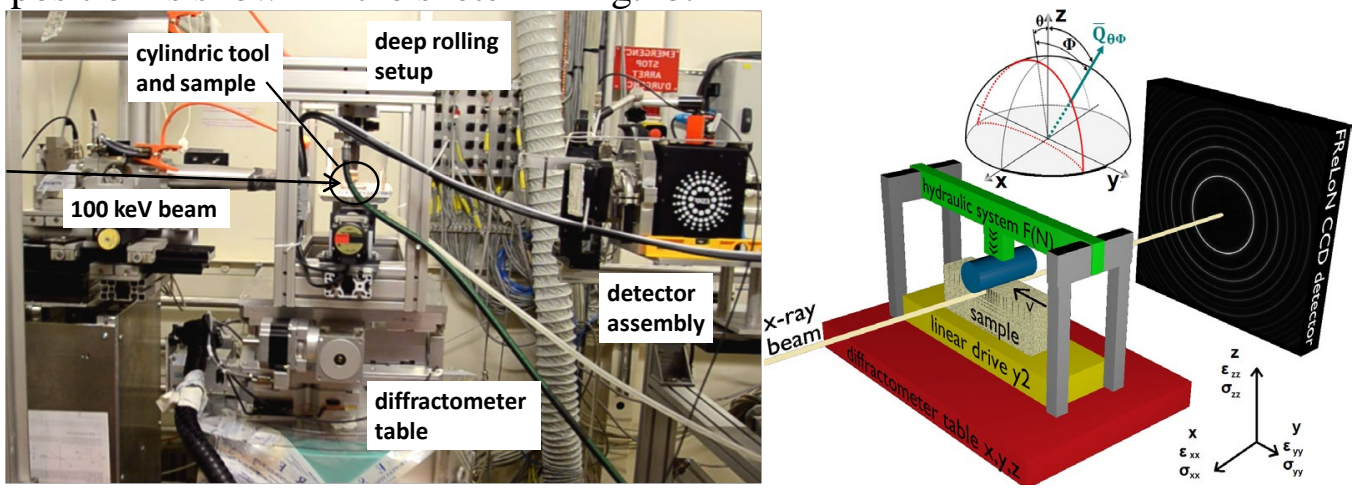

Figure 1. (a) Deep rolling setup installed in beamline ID11 EH3 (b) sketch of system setup and measurement geometry

With the fast-readout camera, full diffraction rings for distributed points inside the measurement window were collected with a $0.05 \times 0.05 \mathrm{~mm}$ beam size at $100 \mathrm{keV}$ photon energy, with increasing spatial resolution to the contact point, allowing a $2 \mathrm{D}$ reconstruction of the material state below the processed surface. The samples are assumed to be isotropic and homogeneous and therefore the samples were moved below the tool during the process, while the scanning was defined in relation to the contact position of the tool. A total of 960 points were measured in a region of $7.8 \mathrm{~mm}$ in width and $3.65 \mathrm{~mm}$ in depth. The measurement method is comparable to the analysis of static indentation with the same experimental setup in [6].

For the specimens, steel AISI 4140 (42CrMo4) was prepared in two heat treatment states, with a quenched and tempered (QT) state with $506 \mathrm{HV} 1$ hardness, a yield strength of $\sigma_{\mathrm{y}}=1440$ 
$\mathrm{MPa}$ and an ultimate tensile strength of $\sigma_{\mathrm{UTS}}=1570 \mathrm{MPa}$, and a comparatively soft ferriticpearlitic (FP) state with $188 \mathrm{HV} 1$ with $\sigma_{\mathrm{y}}=420 \mathrm{MPa}$ and $\sigma_{\mathrm{UTS}}=730 \mathrm{MPa}$.

\section{Experimental methods}

Using the $\alpha\{211\}$ reflex of the material state, strain value was calculated from the diffraction rings for each of the 960 points in the measurement window, using the fundamental equation of strain as given by Noyan and Cohen [7]. It was determined that only the $\varepsilon_{\mathrm{yy}}, \varepsilon_{\mathrm{zz}}$ and $\varepsilon_{\mathrm{yz}}$ strain components in the respective coordinate system have to be analyzed (Eq.1) in a plane strain approach, since the boundary conditions of the specimen only introduce negligible deviations in this mode, as found by Uhlmann et al [5] for a comparable orthogonal cutting process.

$$
\varepsilon_{\theta \delta}(\mathrm{y}, \mathrm{z})=\cos ^{2} \theta \sin ^{2} \phi \varepsilon_{\mathrm{yy}}(\mathrm{y}, \mathrm{z})+\cos ^{2} \theta \cos ^{2} \phi \varepsilon_{\mathrm{zz}}(\mathrm{y}, \mathrm{z})+\cos \theta \sin 2 \phi \varepsilon_{\mathrm{yz}}(\mathrm{y}, \mathrm{z})
$$

A total number of 72 azimuthal sections were integrated from the diffraction rings with each $5^{\circ}$ range, after data treatment with the pyFAI program by Ashiotis et al [8]. These were analyzed for strain and recalculated to stress values on the assumption of plane strain state and using X-Ray elastic constants $1 / 2 \mathrm{~S}_{2}=5.811^{-5} \mathrm{MPa}^{-1}$ for steel specimens [7]. Full-width at halfmaximum (FWHM) was determined as the mean value from pseudo-Voigt peak fits.

The error of stress determination in this method ranges from $100 \mathrm{MPa}$ in the ferritic-pearlitic material to around $50 \mathrm{MPa}$ for each stress component in the tempered martensitic structure. The cause for the difference lies in the much larger grains (crystallites) in the ferritic-pearlitic state, which have a detrimental influence on the grain statistic and therefore on the diffraction signal quality.

As a comparable stress value, von Mises equivalent stress $\sigma_{\text {eq }}$ was used to determine the amount and distribution of equivalent loading and residual stresses in the material with respect to its material state, as introduced by Kuznetsov et al. [9] for burnishing processes.

\section{Equivalent stress and FWHM distribution in the processed material}

The transmission geometry allows for analysis of the material state in a gauge volume comparable to the width of the specimen and the beam cross section. The equivalent stresses in the ferritic-pearlitic and QT specimens are given in Figure 2. The 2D reconstruction of the equivalent stress fields show that the FP state has a wide distribution of the maximum values from the region directly in contact with the roller to the superposition of loading stress and residual stress in the outgoing material. Calculated equivalent stress values are at a maximum of $800 \mathrm{MPa}$ in depth after plastification of the material, which is a value higher than the determined yield strength of the material measured ex-situ. Since the initial material state would allow further deformation at this value, it can be reasoned that work hardening through increased dislocation density allows for an increase of stress state up to the new increased yield strength of the deformed material, as noted by Altenberger [10]. The increased overall equivalent stress in the material at $\mathrm{y}=3.3 \mathrm{~mm}$ can be attributed to residual stresses in the measured components up to a depth of $\mathrm{z}=-2 \mathrm{~mm}$, which reach around $\sigma_{\mathrm{eq}}=400 \mathrm{MPa}\left(\sigma_{\mathrm{yy}}=-70 \mathrm{MPa}\right)$. However, a slight superimposition with the loading stress field is still present at this distance.

For the QT specimen, the higher yield strength leads to a concentration of the loading stress to the region directly under the contact point, with a maximum of $1400 \mathrm{MPa}$ in depth, producing a single drop-shaped influence zone of loading stresses in the material. The measured equivalent stress value is not higher than the yield strength in this case, since work-hardening in this material state cannot strongly shift the elastic limit to higher values. This will also be shown in the following section on FWHM behavior. Residual stress values are comparable to the ferriticpearlitic measurement at around $\sigma_{\mathrm{eq}}=400 \mathrm{MPa}\left(\sigma_{\mathrm{yy}}=-170 \mathrm{MPa}\right)$ in equivalent stress, but at a depth of $0.8 \mathrm{~mm}$ more constrained to the surface near region. 

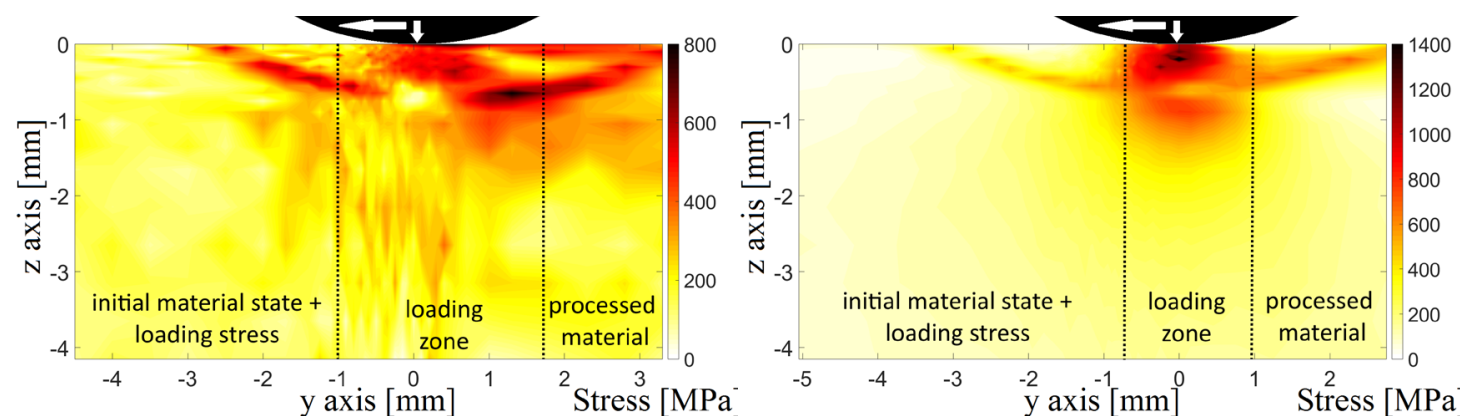

Figure 2. Equivalent stress $\sigma_{\text {eq }}$ distribution at $3400 \mathrm{~N}$ deep rolling contact in (a) ferritic-pearlitic and (b) QT material state

Aside from stress evaluation, the full-width at half maximum (FWHM) of the $\alpha\{211\}$ peaks can be used to evaluate the cold-working introduced into the material, giving a qualitative value for the change in crystallite size, microstrains and dislocation density in the specimen introduced by the deep rolling process. To compare the different initial structures, the change of peak width ( $\triangle \mathrm{FWHM}$ ) compared to the original state was calculated. Corresponding to the effect measured in the stress fields, the difference between the two material states can be clearly found in the higher affected depth of the plastification in the more ductile ferritic-pearlitic state, where an increase of $\triangle F W H M$ can be detected until $2 \mathrm{~mm}$ below the surface (Fig. 3a), which corresponds to the residual stress region in Fig. 2a. This shows that these two modifications are directly connected, as already found in ex-situ measurements by Meyer et al. [1]. A maximum increase of FWHM is detected behind the roller, which then decreases again. FWHM increase caused by plastic deformation should remain constant after unloading. Therefore, the reversible increase in peak width at this position is expected to be connected to a superimposition of inhomogeneous $1^{\text {st }}$ kind stresses within the gauge volume and possibly inhomogeneous $2^{\text {nd }}$ kind stresses depending on the microstructure, with microstrains ( $3^{\text {rd }}$ kind stresses) and crystallite size effects. In the case of FP specimen, only small amount of FWHM increase is reversible, while in QT state most local increase is released after unloading (Fig. 3b). Similar behavior depending on the microstructure were observed in in-situ neutron tensile tests by Tomota et al. and attributed to inhomogeneous $2^{\text {nd }}$ kind stresses between different phases [11]. The temporary change in FWHM from the contact is comparable for both material states at a value of around $1.5 \cdot 10^{-2} \circ$.
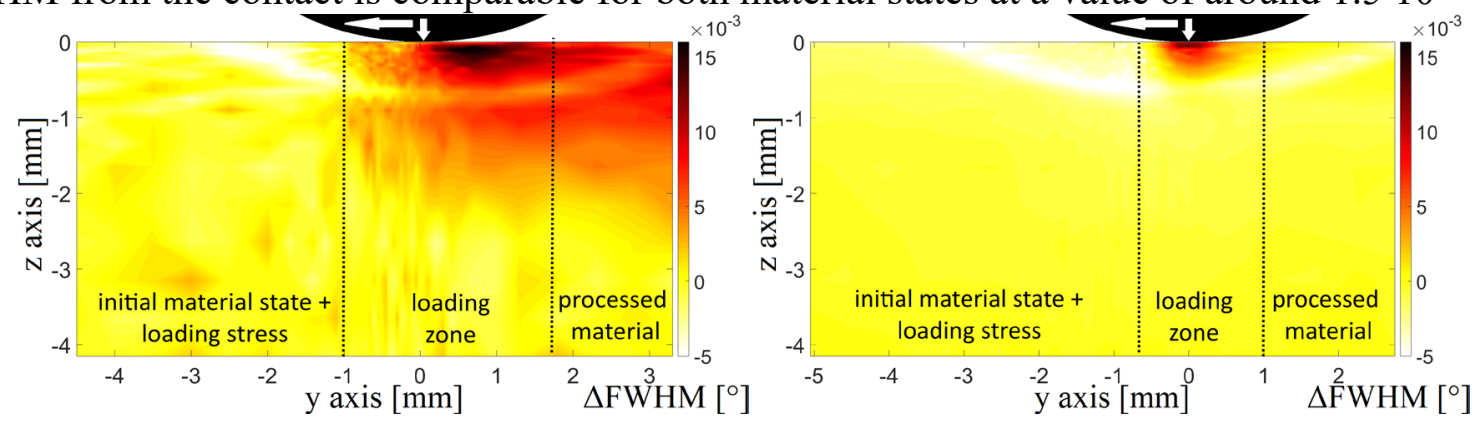

Figure 3. $\triangle F W H M$ distribution at $3400 \mathrm{~N}$ deep rolling contact in (a) ferritic-pearlitic and (b) QT material state

For the QT state (Fig. 3b), a zone with FWHM decrease compared to the initial state (bright region) starting ahead of the tool can be observed. In the treated region behind the tool, this zone is still present. This behavior is already well-known for initial material state with high hardness and high lattice defect density and is due to dislocation reorganization caused by the introduced mechanical energy, causing dislocation walls, tangles and cells in low energy microstructures, as found by Hoffmann et al [12], which leads to reduced measured FWHM values for deep rolled 
QT specimens, as found from ex-situ experiments of this material state and the study of Perenda et al. [13] on hardened deep rolled parts.

\section{Integrated FWHM and stress progression}

Since the effect of deep rolling is restricted to the surface-near region analyzed in this study, the overall effect of stress and FWHM change can be evaluated during the contact sequence if the values are integrated over depth as a function of the y position (Figure 4). For the ferriticpearlitic material, the effect of loading stress can be first detected around $\mathrm{y}=-3 \mathrm{~mm}$ in front of the roller and is increasing continuously from $\sigma_{\mathrm{eq}}=0.6 \cdot 10^{3} \mathrm{MPa} \cdot \mathrm{mm}$, before contact, derived from the residual stress measurement error, to $\sigma_{\text {eq }}=1.2 \cdot 10^{3} \mathrm{MPa} \cdot \mathrm{mm}$ at $\mathrm{y}=0 \mathrm{~mm}$ directly under the contact point. The corresponding increase in peak width from $\Delta \mathrm{FWHM}=0^{\circ} \cdot \mathrm{mm}$ before contact to a maximum value of $\triangle \mathrm{FWHM}=1.5 \cdot 10^{-3}{ }^{\circ} \cdot \mathrm{mm}$ at $\mathrm{y}=0 \mathrm{~mm}$ starts $1.2 \mathrm{~mm}$ later than the beginning of the loading stress increase but both reach maximum values at the same position. Both components than show more or less stable values in the region of remaining material modifications at $\mathrm{y}>2.5 \mathrm{~mm}$, as shown in Fig. 4a. In contrast to this, the QT in Fig. 4b state shows progressive increase in equivalent stresses while the peak width first decreases. This corresponds to the zone of reorganization of dislocation structure due to mechanical energy as described in the previous section. At the position directly below the tool, strong increase of $\Delta$ FWHM take place which can be attributed to superimposition of inhomogeneous $1^{\text {st }}$ and $2^{\text {nd }}$ kind reversible stresses from loading maximum at the same position. After progressive decrease of loading stress influence at $\mathrm{y}>1 \mathrm{~mm}$, the integrated FWHM returns back to value close to 0 , which confirms the already described phenomena. The equivalent stress maximum below the contact point is followed by a decreasing effect of load stress and the generated residual stress state is visible in the asymmetric decrease of the curve. Since stabilization of the evolution is not reached at $\mathrm{y}=3 \mathrm{~mm}$, a slight influence of the load stress is still remaining at this distance.
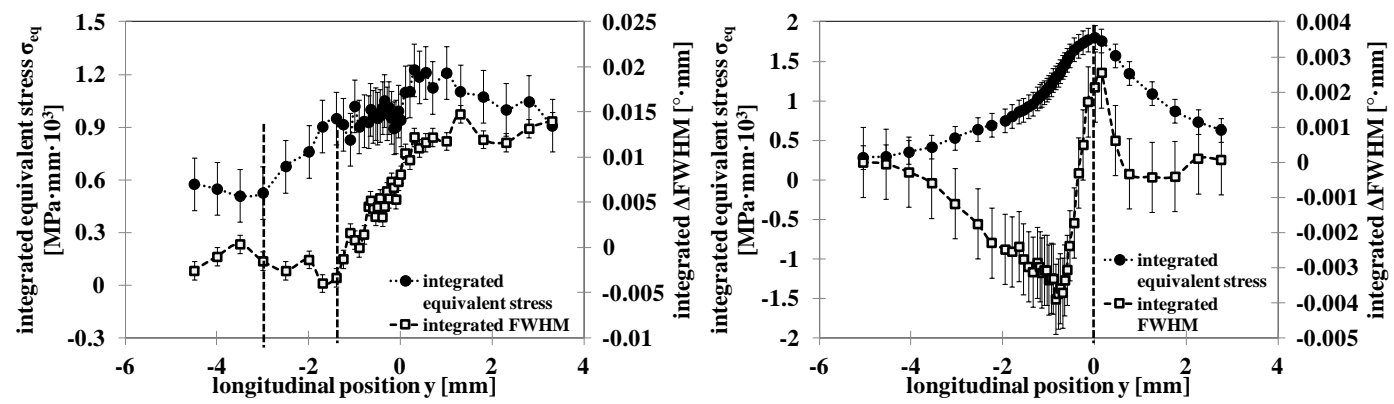

Figure 4. Integrated $\triangle F W H M$ and $\sigma_{\text {eq }}$ over depth in processing direction for (a) ferritic-pearlitic and (b) QT material state

Overall integrated stress state in QT material is higher at around 1.9 MPa'mm compared to 1.2 $\mathrm{MPa} \cdot \mathrm{mm}$ in the softer FP state, which results from the different contact areas and achievable stress state limited by the local yield strength in each case.

\section{Conclusion}

Synchrotron experiments have been performed to analyze the material state of a specimen under deep rolling contact during the process. The local distribution of stresses and peak width could be analyzed during the process, allowing evaluation of loading stresses, generated residual stresses and their superposition in the material. The distribution of stresses is linked to the material properties, where amount and depth of residual stress depends on the yield strength of material. Changes in the width of the analyzed diffraction peaks depend on the initial material state and allowed the evaluation of plastically deformed layer. Since both values show corresponding reactions of the material to the deep rolling contact, an extrapolation of the overall 
material modifications through the tool contact may be possible depending on material properties and contact parameters. With these results, process signature for deep rolling will be further developed.

\section{Acknowledgement}

The funding by the German Research Foundation (Deutsche Forschungsgemeinschaft, DFG) for financial support of subproject C01 within the CRC TRR 136 "Process Signatures" is gratefully acknowledged.

\section{References}

[1] Meyer, D., Kämmler, J. (2016). Surface integrity of AISI 4140 after deep rolling with varied external and internal loads. Procedia CIRP, 45, 363-366. https://doi.org/10.1016/j.procir.2016.02.356 [2] Nikitin, I., \& Altenberger, I. (2007). Comparison of the fatigue behavior and residual stress stability of laser-shock peened and deep rolled austenitic stainless steel AISI 304 in the temperature range 25-600 C. Materials Science and Engineering: A, 465(1-2), 176-182.

https://doi.org/10.1016/j.msea.2007.02.004

[3] Sayahi, M., Sghaier, S., Belhadjsalah, H. (2013). Finite element analysis of ball burnishing process: comparisons between numerical results and experiments. International Journal of Advanced Manufacturing Technology, 67(5-8), 1665-1673. https://doi.org/10.1007/s00170-012-4599-9

[4] Brinksmeier, E., Klocke, F., Lucca, D. A., Sölter, J., Meyer, D. (2014). Process Signatures-a new approach to solve the inverse surface integrity problem in machining processes. Procedia CIRP, 13, 429-434. https://doi.org/10.1016/j.procir.2014.04.073

[5] Uhlmann, E., Henze, S., Gerstenberger, R., Brömmelhoff, K., Reimers, W., Fischer, T., Schell, N. (2013). An extended shear angle model derived from in situ strain measurements during orthogonal cutting. Production engineering, 7(4), 401-408. https://doi.org/10.1007/s11740-013-0471-5

[6] Meyer, H., Epp, J., Zoch, H.W., (2017). In situ X-Ray Diffraction Investigation of Surface Modifications in a Deep Rolling Process under Static Condition. Materials Research Proceedings, 2, 431-436. https://doi.org/10.21741/9781945291173-73

[7] Noyan, I. C., Cohen, J. B. (2013). Residual stress: measurement by diffraction and interpretation. Springer.

[8] Ashiotis, G., Deschildre, A., Nawaz, Z., Wright, J. P., Karkoulis, D., Picca, F. E., Kieffer, J. (2015). The fast azimuthal integration Python library: pyFAI. Journal of applied crystallography, 48(2), 510-519. https://doi.org/10.1107/S1600576715004306

[9] Kuznetsov, V. P., Smolin, I. Y., Dmitriev, A. I., Konovalov, D. A., Makarov, A. V., Kiryakov, A. E., Yurovskikh, A. S. (2013). Finite element simulation of nanostructuring burnishing. Physical Mesomechanics, 16(1), 62-72. https://doi.org/10.1134/S1029959913010074

[10] Altenberger, I. (2005, September). Deep rolling - the past, the present and the future. Proceedings of 9th international conference on shot peening, 6-9.

[11] Tomota, Y., Lukáš, P., Neov, D., Harjo, S., \& Abe, Y. R. (2003). In situ neutron diffraction during tensile deformation of a ferrite-cementite steel. Acta materialia, 51(3), 805-817. https://doi.org/10.1016/S1359-6454(02)00472-X

[12] Hoffmann, B., Vöhringer, O., Macherauch, E. (2001). Effect of compressive plastic deformation on mean lattice strains, dislocation densities and flow stresses of martensitically hardened steels. Materials Science and Engineering: A, 319, 299-303. https://doi.org/10.1016/S09215093(01)00978-9

[13] Perenda, J., Trajkovski, J., Žerovnik, A., Prebil, I. (2015). Residual stresses after deep rolling of a torsion bar made from high strength steel. Journal of Materials Processing Technology, 218, 89-98. https://doi.org/10.1016/j.jmatprotec.2014.11.042 